\title{
A review of the New Geophysics: a new understanding of pre-fracturing deformation in the crack-critical crust with implications for hydrocarbon production
}

\author{
Stuart Crampin ${ }^{1,2,3 *}$ and Yuan Gao Ga, $^{3,1}$ \\ ${ }^{1}$ School of GeoSciences, University of Edinburgh, UK \\ ${ }^{2}$ Edinburgh Anisotropy Project, British Geological Survey, Edinburgh, UK \\ ${ }^{3}$ Institute of Earthquake Science, China Earthquake Administration, Beijing, China
}

\begin{abstract}
This paper reviews a new understanding of shear-wave splitting (seismic-birefringence) that is a fundamental revision of conventional fluid-rock deformation. It is a New Geophysics with implications for almost all solid-earth geosciences, including hydrocarbon exploration and production, and earthquake forecasting. Widespread observations of shear-wave splitting show that deformation in in situ rocks is controlled by stress-aligned fluid-saturated grain-boundary cracks and preferentially orientated pores and pore-throats pervasive in almost all igneous, metamorphic, and sedimentary rocks in the Earth's crust. These fluid-saturated microcracks are the most compliant elements of the rock-mass and control rock deformation. The degree of splitting shows that the microcracks in almost all rocks are so closely spaced that they verge on fracture-criticality and failure by fracturing, and are critical systems with the "butterfly wing's" sensitivity of all critical systems. As a result of this crack-criticality, evolution of fluid-saturated stress-aligned microcracked rock under changing conditions can be modelled with anisotropic poroelasticity (APE). Consequently, low-level deformation can be: monitored with shear-wave splitting; future behaviour calculated with APE; future behaviour predicted with APE, if the change in conditions can be quantified; and in principle, future behaviour controlled by feed-back. This paper reviews our current understanding of the New Geophysics of low-level pre-fracturing deformation.
\end{abstract}

Key words: Crack anisotropy, New Geophysics, pre-fracturing deformation, seismic birefringence, shear-wave splitting

\section{Introduction}

Worldwide observations of stress-aligned shear-wave splitting (seismic birefringence) in hydro-carbon reservoirs and above small earthquakes show that almost all in-situ rocks are pervaded by distributions of stress-aligned fluidsaturated microcracks (Crampin, 1994; 1999). These are grain-boundary cracks in crystalline rocks, and preferentiallyaligned pores and pore-throats in sedimentary rocks. The degree $(\%)$ of observed shear-wave velocity anisotropy shows that microcracks in in situ rocks are so closely-spaced they verge on fracture criticality, when levels of cracking are so great that shear-strength is lost and failure by fracturing is likely (Crampin, 1994; 1999). Phenomena verging on criticality in this way are critical systems (Davis, 1989) with self-organised criticality (Jensen, 1998).

Critical systems are a New Physics (Davis, 1989), in our case a New Geophysics, with fundamentally different

*Corresponding author. email: scrampin@ed.ac.uk; Papers and preprints at www.geos.ed.ac.uk/homes/scrampin/opinion Received September 10, 2007 properties from conventional sub-critical behaviour. Below criticality, individual elements of the system, the fluidsaturated microcracks in the Earth's crust, interact locally and heterogeneously with conventional well-understood behaviour. The behaviour can be calculated but the final position may depend critically on minuscule differences in initial conditions. At criticality, the elements throughout the region abruptly act in unison and rocks, sometimes over very large volumes, spontaneously fracture with deterministic chaos (Bruce and Wallace, 1989). "It is one of the universal miracles of nature that huge assemblages of particles, subject only to the blind forces of nature, are nevertheless capable of organising themselves into patterns of cooperative activity" (Davis, 1989). Such behaviour at criticality is characteristic of almost all complex interactive systems which are extremely common. Thus the claim that the cracks in the crust are critical systems merely suggests that the Earth behaves like all other complex interactive systems: the weather; quantum mechanics; life cycle of fruit flies; and clustering of traffic on roads; and a huge range of physical phenomena (Davis, 1989; Crampin and Chastin, 2003).

Note that undisturbed in situ rocks are wholly inaccessible 
and we must gather information from all available sources. Consequently, this review assesses evidence from both exploration and earthquake seismics. Earthquake seismics is briefly discussed in the Appendix.

\section{Shear-wave splitting}

The key phenomenon for observing the internal microcracked structure and deformation of in situ rocks is stress-aligned shear-wave splitting. In anisotropic rocks, shear-waves split into two approximately orthogonal polarisations where the faster polarisation is typically aligned in the direction of the maximum horizontal stress (Fig. 1) (Crampin, 1994; 1999). Stress-aligned shear-wave splitting is a second-order effect that, by rotating horizontal seismograms into preferential orientations, can typically be measured with first-order accuracy, where the parameters that control shear-wave splitting are directly those that control low-level deformation before fracturing occurs (Zatsepin and Crampin, 1997; Crampin and Zatsepin, 1997).

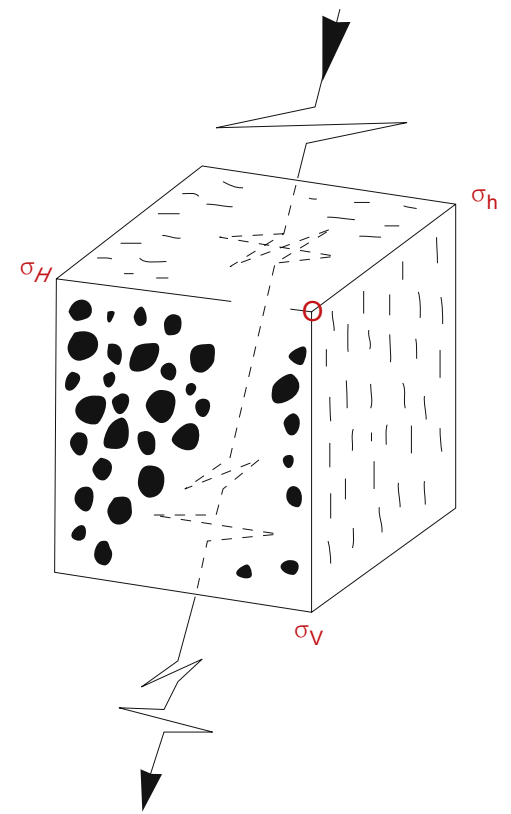

Fig. 1 Schematic illustration of stress-aligned seismic shear-wave splitting in fluid-saturated stress-aligned microcracked rock in crustal rock

Note that although shear-wave splitting is comparatively simple in concept, in practice there are several subtleties which may complicate interpretation. The reader is referred to Crampin (1981) and Crampin and Peacock (2005) for an outline of anisotropic wave propagation in cracked rocks.

\subsection{Confirmation of stress-aligned fluid-saturated cracks in the crust}

Observations of shear-wave splitting at the free-surface typically display polarisations aligned approximately parallel to the direction of maximum horizontal stress in reflection profiles and Vertical seismic profiles (VSPs) in the seismic exploration (Winterstein et al, 2001; Angerer et al, 2002), and above small earthquakes (Crampin, 1981; Gao et al, 1998; Volti and Crampin, 2003b). The only anisotropic symmetry system that has such parallel polarisations for a large part of the shear-wave window is transverse-isotropy with a horizontal axis of symmetry, or a minor variation thereof. This symmetry is frequently known as HTI- or TIH-anisotropy. Moreover, the only common geological configuration that has such HTI-anisotropy is distributions of fluid-saturated microcracks aligned perpendicular at the direction of minimum horizontal stress which is typically horizontal. Fluid-saturated microcracks are confirmed as the source of the splitting, as only microcracks have sufficient compliance to respond to changing fluid pore-pressures in oil reservoirs (Angerer et al, 2002) and small changes in the stress-accumulation before earthquakes (Gao et al, 1998; Crampin et al, 1999) (see Appendix). These phenomena demonstrate that stress-aligned shear-wave splitting is typically caused by stress-aligned fluid-saturated microcracks illustrated schematically in Fig. 1.

Note that the exceptional rocks which do not display azimuthally-varying shear-wave splitting are finely layered substrates, and shales, clays, and mudstones with lithological transverse-isotropy which show radially-polarised shearwave splitting. Such rocks have pronounced transverseisotropy with a vertical axis of symmetry known VTI- or TIV-anisotropy. VTI-anisotropy is important in seismic exploration as it is difficult to match horizontal and vertical $P$-wave velocities (unbalanced move-out velocities), but it conveys little geophysical information except that gravity is vertical.

\subsection{Crack density}

Crack density $\varepsilon$ is a dimensionless parameter defined as $\varepsilon=N a^{3} / v$, where $N$ is the number of cracks of radius $a$ in volume $v$ (Crampin, 1994). As crack density, $\varepsilon$, is approximately equal to one hundredth of the percentage of maximum shear-wave velocity anisotropy in any cracked rock (when the Poisson's ratio of the matrix rock is 0.25 ), it is comparatively easy to estimate crack density from observations of vertically propagating shear-wave splitting. (Cracks are typically aligned parallel and vertical perpendicular to the usually-horizontal direction of minimum compressional stress, so that vertical propagation usually shows the maximum shear-wave velocity anisotropy.) Crack density largely determines the overall effect of cracks on the effective elastic tensor of an anisotropic microcracked solid (Crampin and Chastin, 2003; Crampin and Peacock, 2005).

\subsection{Observed range of shear-wave velocity in ostensibly-intact rock}

A range of $1.5 \%$ to $4.5 \%$ shear-wave velocity anisotropy is typically observed in unfractured rock in the Earth's crust (Crampin, 1994, 1999). This is illustrated schematically in Fig. 2, where observations indicate a narrow range $0.015 \leq \varepsilon$ $\leq 0.045$ of inferred crack density in ostensibly intact in situ rock (Crampin and Zatsepin, 1997; Crampin, 1999). These values of shear-wave velocity anisotropy appear to be moreor-less independent of porosity, geology, and tectonic history, and are observed to be similar in all sedimentary, igneous, and metamorphic rocks, with only a few well-understood 


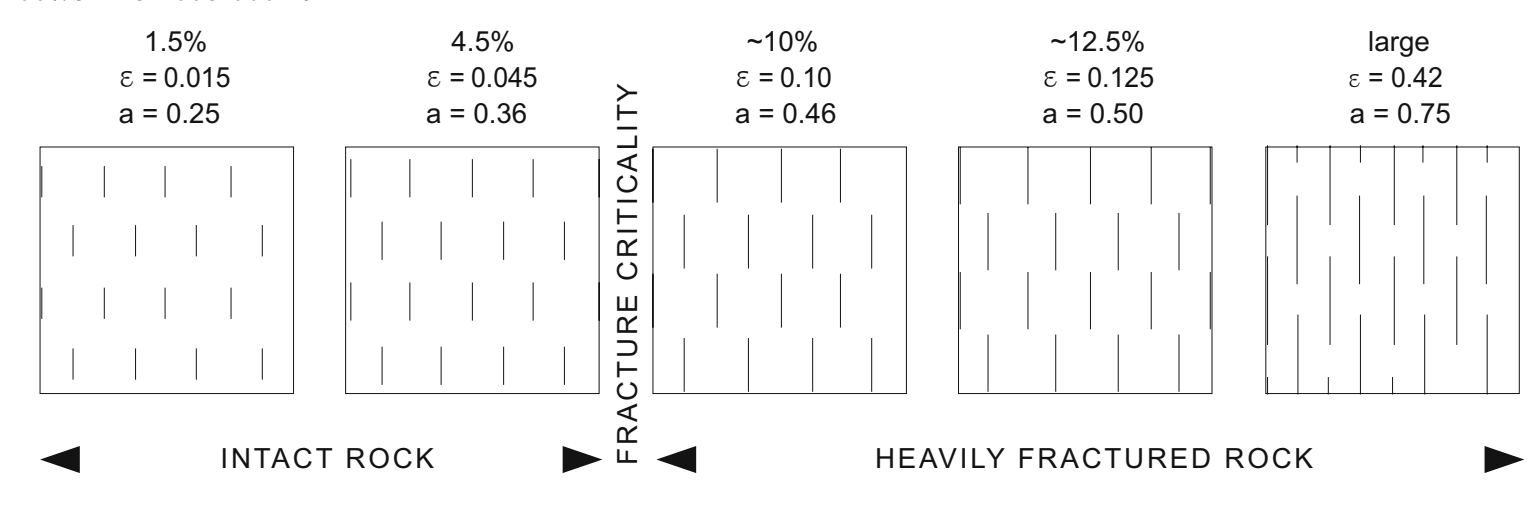

Fig. 2 Schematic illustration of observed percentage of shear-wave velocity anisotropy in terms of uniform distributions of pennyshaped cracks (After Crampin, 1994)

exceptions (Crampin, 1994, 1999). Fig. 2 shows that there is a factor of less than two in average crack radius, between the minimum observed, and the maximum at fracture criticality. This demonstrates that almost all in-situ rocks in unfractured rocks are so heavily microcracked that shear strength lost and fracturing occurs in the presence of almost any disturbance. Thus almost all rocks verge on fracture criticality and failure by fracturing and earthquakes. We associate fracture criticality with the percolation threshold when there are through-going fractures, which for parallel cracks is approximately $\varepsilon=$ $\sim 0.055$ ( $\sim 5.5 \%$ shear-wave velocity anisotropy) (Crampin and Zatsepin, 1997).

Such verging on criticality is the key observation that indicates that fluid-saturated microcracked rocks are critical systems. The universality of critical systems is the reason why the narrow range of $1.5 \%$ to $4.5 \%$ shear-wave velocity anisotropy is observed to pervade almost all in situ rocks independent of rock type, tectonic history, stress-regime, and porosity - the same narrow range is observed in $1 \%$ porosity crystalline rocks as in $30 \%$ porosity sandstones.

\subsection{Shear-wave window}

Shear-waves observed at the surface are sensitive to the angle of incidence to the free-surface. For angles of incidence less than $\sin -1(\mathrm{Vs} / \mathrm{Vp})$, which is $\sim 35$ o for a Poisson's ratio of 0.25 , the incoming shear-wave is accurately recorded (with double the amplitude) within the shear-wave window (Booth and Crampin, 1985). SV-arrivals outside this window are so severely distorted by S-to-P conversions that the direct shear-wave arrival times will not be visible. SH-arrivals are not distorted, but since shear-waves, particularly shear-wave splitting, typically contain combinations of both SV- and $\mathrm{SH}$-waves, all shear-waves recorded outside the shear-wave window are highly unreliable.

Note that near-surface rocks typically have low-velocity layers, due to weathering and stress-release anomalies. Consequently, ray paths tend to curve upwards and the effective shear-wave window, above small earthquakes, say, may frequently be extended to straight-line ray paths with incidence of 450 to $50 \mathrm{o}$.

Note that the shear-wave window is defined with respect to the angle of incidence to the free-surface, not the angle to the horizontal. Consequently, irregular surface topography severely disturbs recordings of shear-waves and shear-wave splitting.

\section{APE model of rock mass evolution}

The evolution of fluid-saturated microcracks in changing conditions can be modelled by Anisotropic Poro-Elasticity (APE) (Zatsepin and Crampin, 1997; Crampin and Zatsepin, 1997). The mechanism for pre-fracturing deformation is fluid movement by flow or dispersion along pressure gradients between neighbouring grain-boundary cracks in crystalline rocks, and aligned pores and pore-throats in sedimentary rocks, at different orientations to the stress field. APE is a fully three-dimensional model but 3D-variations are difficult to plot on 2D-paper, and Fig. 3 shows schematic evolution under increasing differential stress, $\mathrm{sH}$, in horizontal cuts of vertically oriented crack distributions. Hexagons are elastically isotropic, so the initial configuration of solid hexagons with zero differential stress (Fig.3, top left), is a small selection of randomly-oriented vertical cracks.

Under zero differential stress, $\mathrm{sH}=0$, Fig. 3, top left, all cracks have equal aspect-ratios and the geometry is elastically isotropic. With a small increase of differential stress, top right, some fluids move along pressure-gradients between cracks to balance the stress field, and aspect-ratios vary, but as no cracks close there is still no effective anisotropy. When differential stress increases to a critical stress, normalised to $\mathrm{sH}=1$, bottom left, cracks normal to the stress direction begin to close and the isotropy immediately becomes anisotropic. The anisotropy jumps from zero to approximately the same shear-wave velocity anisotropy as the minimum observed in the Earth (compare bottom left image in Fig. 3 with left-hand image in Fig. 2). Thus APE models the observed minimum shear-wave velocity anisotropy which was initially difficult to understand. As the differential stress increases, bottom right, cracks continue to align themselves parallel to the stress field until they reach fracture-criticality at the percolation threshold. There is no fluid leakage and $\sim 6 \%$ porosity is preserved throughout. Thus APE approximately models the observed range of shear-wave velocity anisotropy in unfractured rock.

Note that APE models low-level deformation below levels at which failure occurs. Once the critical-system has reached fracture-criticality, there is failure, fracturing, and 

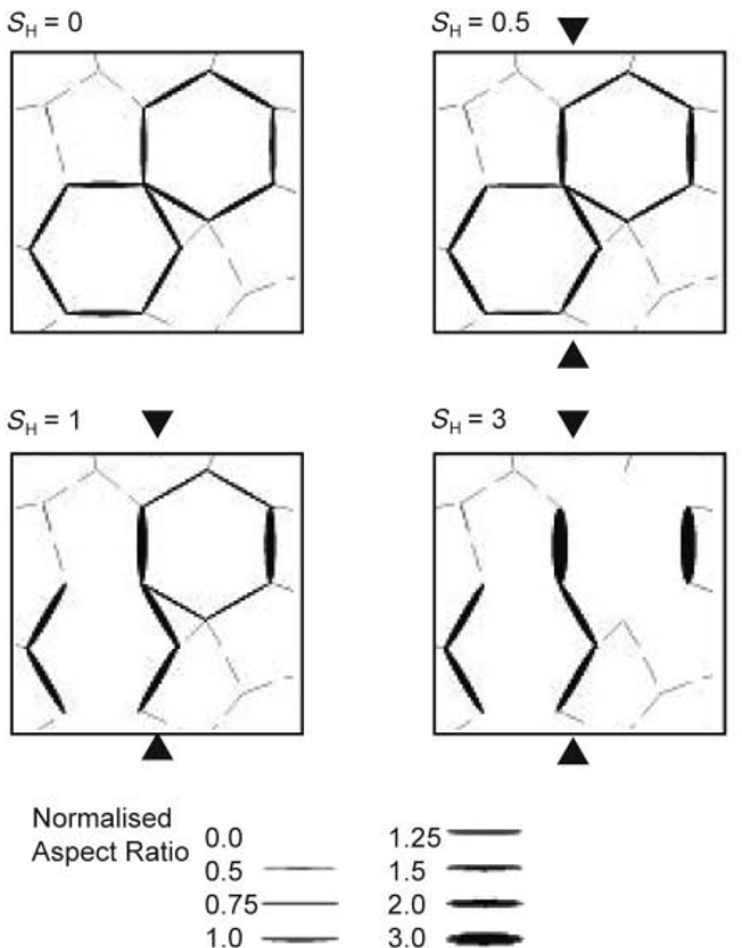

Fig. 3 Schematic image of the Anisotropic Poro-Elastic (APE) model of rock deformation where the driving mechanism is driven by pore-fluid movement along pressure-gradients between neighbouring cracks at different orientations to the stress field (After Crampin and Chastin, 2003)

deterministic chaos. The process of fracturing may be capable of being modelled, but is unrepeatable as the behaviour depends critically on minuscule differences in the initial conditions.

Note also that crack density does not vary consistently with stress accumulation. There are more cracks (higher crack density) at zero differential stress, Fig. 3, top left, than there are when cracks begin to close, Fig. 3, bottom images. The distinguishing feature for recognising increasing low-level stress is that the average aspect-ratios increase. Increases of average aspect-ratios can be recognized by comparatively subtle variations in the $3 \mathrm{D}$ propagation of shear-wave time delays, as has been observed in the stress-accumulation before earthquakes (Appendix).

It is interesting that as shear-wave splitting is observed whenever appropriate source-recorder geometry exists, Fig. 3 suggests that there is always sufficient differential stress in the Earth's crust to close some stress-normal microcracks and cause shear-wave splitting.

APE-modelling approximately matches a huge range of different phenomena referring to cracks, stress, and shearwave splitting in both exploration and earthquake seismology (Crampin, 1999; Crampin and Chastin, 2003; Crampin and Peacock, 2005). The match can be only approximate because the internal microcrack structure of in-situ rocks is essential inaccessible and impossible to monitor reliably. Even drilling thin boreholes, locally deforms in situ rock by de-stressing, cooling, and disturbing the compliant internal microcrack geometry by invasion of high-pressure drilling fluids.

\subsection{Response of shear-wave splitting to changes of stress and pressure}

APE-modelling shows in Fig. 3 that the principal effect of changes of low-level stress and pore-fluid pressure is to modify the microcrack aspect-ratios of cracks aligned in the direction of maximum horizontal stress (Crampin and Zatsepin, 1997; Crampin, 1999). In particular, increases of low-level stress before fracturing occurs (pre-fracturing deformation), as in the stress-accumulation before earthquakes (see references in Appendix), can be monitored by increases in the average shear-wave splitting time-delays in Band- 1 of the shear-wave window. Band-1 is the doubleleafed solid-angle segment making angles between $15^{\circ}$ and 450 to the normal of the distribution of vertical parallel cracks (Crampin, 1999). Observations of such, rather subtle, easily overlooked, changes monitor the accumulation of stress before earthquakes and allow the progress towards fracture-criticality and earthquake occurrence to be estimated (see Appendix). In appropriate circumstances this can lead to successful forecasting of the time, magnitude, and location of impending earthquakes (Crampin et al, 1999).

Note that changing crack-density modifies time-delays in Band-2 of the shear-wave window, where Band-2 is the solidangle $\pm 15^{\circ}$ either side of the plane of the crack distributions. As suggested above, crack density does not have any simple relationship with low-level changes of stress.

The most successful direct in-situ calibration of APE to-date (Fig. 4) modelled changes in three-component reflection-surveys before and after a high-pressure, 6.4 $\mathrm{MPa}$, $\mathrm{CO}_{2}$-injection into a carbonate reservoir at $1600 \mathrm{~m}$ depth in Vacuum Field, New Mexico (Angerer et al, 2002). The match of the APE-model to observations is excellent, as it also was for a low-pressure, $2 \mathrm{MPa}$, injection (not shown). These are the best in-situ demonstrations to-date of the application of APE-modelling. It suggests that, when changes to the rock mass are known, the effects can be calculated by APE, at least in some circumstances, and behaviour predicted if the changing conditions can be quantified. It also means that if a particular result is desired, a particular degree of aligned cracking during a water-flood operation, say, the intended effect can be controlled by feed-back by adjusting fluidpressure injections.

\section{$3.290^{\circ}$-flips in shear-wave polarisations in high- pressure crack fluids}

Fig. 4 also shows another confirmation of APE-modelling. In Fig. 4(a) the two-way reflection times of the (faster) S1wave from the top and bottom of the target zone differ by $176 \mathrm{~ms}$ whereas, as expected, the delay for the (slower) S2wave is larger, $178 \mathrm{~ms}$. In contrast, in Fig. 4(b) after the critically-high-pressure injection, with the same seismogram rotations, the $S 1$-wave delay is $204 \mathrm{~ms}$ and the $S 2$-wave delay is $184 \mathrm{~ms}$. This means that the slower and faster split shearwaves have exchanged polarisations. There has been a $90^{\circ}$ -flip in shear-wave polarisations in the presence of criticallyhigh pore-fluid pressures (Angerer et al, 2002; Crampin 

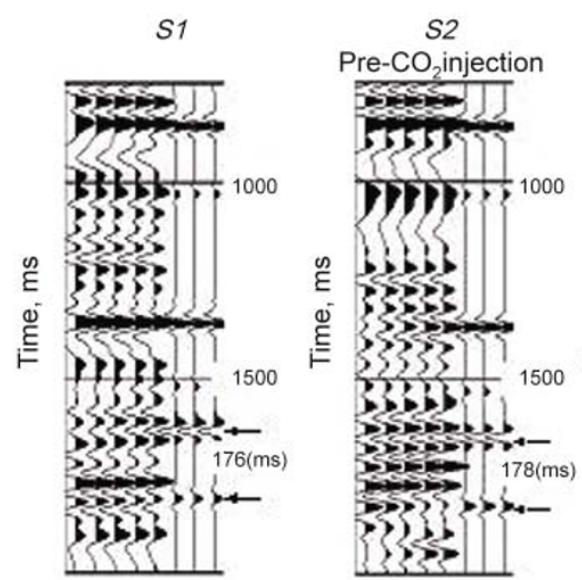

(a)

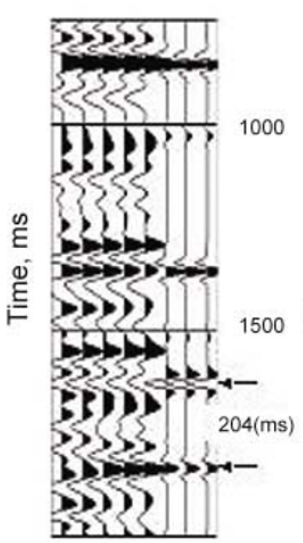

Post-CO injection

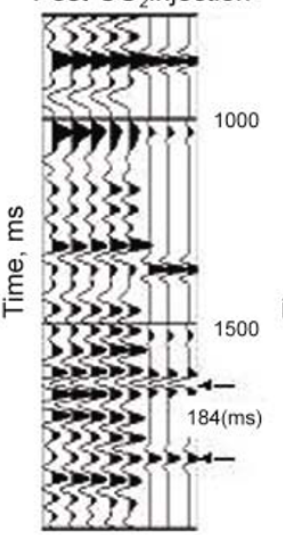

(b)

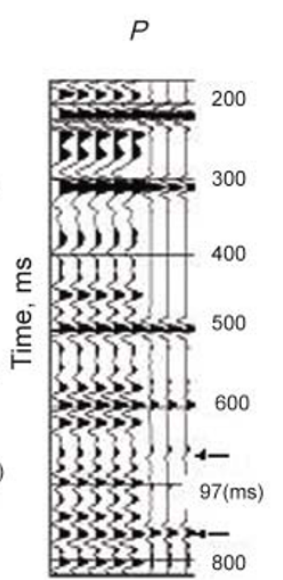

is 35$\}$ it 800

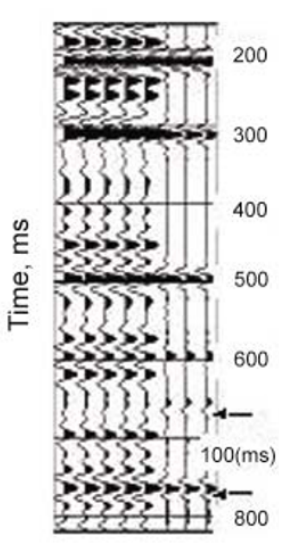

Fig. 4 Modelling changes in shear-wave splitting in reflection surveys before and after a critically high-pressure $\mathrm{CO}_{2}$-injection in Vacuum Field, New Mexico, a fractured carbonate reservoir. (a) Pre-injection waveforms of a multi-component nearly-vertical ray reflection survey near the centre. The record section are rotated into mutually orthogonal S1, S2, and P polarisations, where the horizontals $\mathrm{S} 1$, and $\mathrm{S} 2$, have been rotated into the split shear-wave polarisations parallel and perpendicular to the direction of maximum horizontal stress, respectively. The five left-hand-side traces are observed waveforms at recorders $17 \mathrm{~m}$ apart, and the three right-handside traces are synthetic seismograms modelled by APE matching the shearwave arrivals. (b) Similar traces following high-pressure $\mathrm{CO}_{2}$-injection where exact pressure increases were modelled by APE (After Angerer et al., 2002)

et al, 2002). These $90^{\circ}$-flips, in some circumstances can lead to observations of time-delays between split shearwaves decreasing with distance (Crampin et al, 1996), and observations of shear-wave polarisations parallel to the minimum horizontal stress instead of the more typical parallel to the maximum horizontal stress (Crampin et al, 2002).

$90^{\circ}$-flips, initially modelled theoretically by APE (Crampin and Zatsepin, 1997), have now been recognised in critically high-pressured hydrocarbon reservoirs (Crampin et al, 1996; and Fig. 4, Angerer et al, 2002). $90^{\circ}$-flips are also recognised above all seismically-active fault zones due to the criticallyhigh pore-fluid-pressures necessary to permit movement on lithostatically compressed faults (Crampin et al, 2002).

Note that $90^{\circ}$-flips occur in response to critically-high pore-fluid pressures whenever pore-pressures approach the maximum horizontal stress, $\mathrm{sH}$. Conventional over-pressures in the oil industry, when pore-pressure exceeds the hydrostatic

stress, are not usually high-enough to cause $90^{\circ}$-flips.

\section{Critical-systems of fluid-saturated cracks in the earth}

Critical-systems are typically sensitive (with "butterfly wing 's" sensitivity) to otherwise negligible variations in initial conditions that can lead to order-of-magnitude differences as the systems evolve (Davis, 1989; Bruce and Wallace, 1989; Crampin and Chastin, 2003). The manipulation of fluidsaturated microcracks as monitored by shear-wave splitting indicates that stress-aligned fluid-saturated microcracks are remarkably pervasive features with similar parameters in almost all rocks in the crust. The observed calculability, modelled in Fig. 4, say, is because the fluid-saturated cracks in the crust are so closely spaced that they are critical systems. Critical systems involve dynamic interactive processes that below criticality perturb only locally, whereas once systems reach criticality, all members of the system influence all other members and the behaviour is collaborative.

Similar schemes for the Earth have been suggested previously. The difference here is that we have now identified the micro-scale mechanism for deformation as stress-induced fluid-movement along pressure-gradients between adjacent fluid-saturated grain-boundary cracks and pore-throats. This is a quantifiable physical process that can be modelled, monitored, calculated, and in principle predicted, even controlled by feedback using APE.

The criticality, where all critical systems have similar statistical behaviour, despite very different sub-critical physics, is the reason APE-modelling matches a huge range of phenomena (Crampin and Chastin, 2003; Crampin, 2006). This is known as critical-point universality (Bruce and Wallace, 1989), and implies the self-similar scaling seen in crack distributions, as well as the well-known GutenbergRichter earthquake magnitude frequency relationship, and an ever increasing range of other phenomena (Crampin and Peacock, 2005; Gao and Crampin, 2006). Self-similar scaling is where variations are straight lines when plotted in logarithmic axes.

\subsection{Direct evidence of rock sensitivity: the prototype stress-monitoring site}

The expected sensitivity and compliance of the New Geophysics has been directly confirmed by a cross-hole experiment in a prototype borehole Stress-Monitoring Site (SMS) for stress-forecasting earthquakes (Crampin, 2001). The "SMSITES" SMS near the Húsavík-Flatey transform fault of the Mid-Atlantic Ridge in Northern Iceland recorded horizontal seismic travel times at $500 \mathrm{~m}$-depth between boreholes offset 315 m (Crampin et al., 2003). Repeated pulsing of a Downhole Orbital Vibrator (DOV) (Leary et al., 2003; Walter et al., 2003) borehole shear-wave source several times each minute for 13 days allowed, with 100 -fold stacking, $\pm 0.02 \mathrm{~ms}$ time-lapse accuracy in P-wave and split shear-wave ( $S H$ and $S V$ ) travel times and shearwave velocity anisotropy $(S V-S H)$. The arrivals showed wellrecorded $5 \%, 2 \%, 2 \%$, and $10 \%$ variations, respectively, which correlated with NS and EW Global Positioning System (GPS) 
measurements, and an abrupt five-day one-metre decrease in water-level in a water-well immediately above the Húsavík-Flatey fault.

All seven measurements in Fig. 5 coincided with smallscale seismicity, with equivalent energy release to one $M 3.5$ earthquake, on a neighbouring transform fault $70 \mathrm{~km} \mathrm{NNW}$ of SMSITES. These well-recorded variations show exceptional sensitivity to the stress-release of comparatively small earthquake energy at several hundred times the conventional earthquake-source diameter, and well beyond that expected in a conventional brittle-elastic crust (Crampin et al, 2003). This confirms the exceptional sensitivity of a critical-system of fluid-saturated stress-aligned microcracks.

\subsection{Indirect evidence of rock sensitivity}

The Appendix reports observations of stress-accumulation have been monitored (in retrospect) at before some 15 earthquakes ( $m b 1.7$ to $M s 7.7$ ) with one successful stressforecast in real-time. These earthquakes have been in eight widely differing geological and tectonic locations worldwide which suggests that due to the crack-criticality the sensitivity is common to all geological and tectonic regions.

\section{Properties of critical systems of cracks in the crust}

It has been shown that critical systems near criticality (such as microcracks in the crust in Figs. 1, 2 and 3) behave statistically more like other critical systems than the subcritical physics (Bruce and Wallace, 1989; Zatsepin and Crampin, 1997; Crampin and Zatsepin, 1997). This is the reason why the nearly parameterless APE-modelling approximately matches the behaviour of extremely complicated heterogeneous crustal rocks (Crampin and Chastin, 2003; Crampin, 2003; 2006). This means that in practice the behaviour of the anomalies in Fig. 5, for example, may not be explicable or even understandable in terms of conventional sub-critical geophysics. Certainly, critical systems are known to have several remarkable properties (Crampin, 2003; 2006).

Some of these new properties have serious disadvantages for conventional oil-field geophysics and conventional oilfield operations.

D1) The detailed behaviour of reservoirs is temporally and spatially unstable so that any given measurement may degrade in time, as in Fig. 5, where measurements vary significantly with time (and place - not shown).

D2) Many properties of reservoirs are self-similar and are temporally and spatially heterogeneous at all scale lengths. This means that averaging in time and space may only be valid in particular (limited) circumstances.

D3) There is the possibility of long-range interactions between, and long-term interactions within, hydrocarbon reservoirs. The effects of D3 have been observed in large mature oil fields (Heffer et al., 1995).

D4) Detailed behaviour (such as the behaviour in Fig. 5) may not correspond to or be explicable in terms of conventional geophysics.

Despite these complications and disadvantages, there are other properties which are likely to have enormous potential benefits for hydrocarbon recovery.

B1) Current configurations of crack geometry within the deep interior of the rock mass or reservoir can be monitored with shear-wave splitting and evaluated by APE (Crampin, 2003; 2006; Crampin and Chastin, 2003; Crampin and Zatsepin, 1997).

B2) Response of rock mass or reservoir to known changes can be calculated by APE (Zatsepin and Crampin, 1997; Crampin and Zatsepin, 1997; Angerer et al., 2002; etc).

B3) Response of rock mass or reservoir can be controlled by feedback by repeating items B1 and B2. If the response can be calculated and monitored by shear-wave splitting, in principle, the optimal response and optimal recovery can be controlled by adjusting input parameters via feedback.

B4) An important additional constraint is that modelling by APE (items B1 and B2) will only apply to slowly driven systems, when stress relaxation processes and fluid percolation are allowed to evolve naturally (Davis, 1989; Jensen, 1998; Crampin and Chastin, 2003). This leads to the benefit of SMORE: Slower production for More Oil REcovery (Section 6.2) (Crampin, 2006).

\section{Main disadvantages and benefits for hydrocarbon production}

Unquestionably, the biggest operational disadvantages are D1 and D2, and the implication of B4 that reservoirs will only behave predictably if slowly driven and stress is allowed to relax between successive operations .

Evidence for D1, Temporal and spatial instability, is demonstrated directly by the variations in Fig. 5, where repeated measurements between fixed source and fixed receiver vary with time correlating with small distant seismic disturbances. Other evidence (Crampin and Chastin, 2003; and references in the Appendix) suggests these variations could vary from weeks before small earthquakes within tens of $\mathrm{km}$ to years before the largest earthquakes and eruptions that could be visible worldwide.

Other sources of disturbance could be production operations in neighbouring, not necessarily adjacent, oil fields (Heffer et al., 1995), earth tides, and other phenomena. APE implies that the Earth's crust is in a continual state of flux. Consequently, detailed seismic measurements within a reservoir may vary with time due to what are likely to be real but unidentifiable phenomena. These variations are likely to be small but could well contaminate detailed time-lapse (4D) seismic monitoring in production reservoirs (Crampin, 2004).

D2: Temporal and spatial heterogeneities are potentially more serious. The principal heterogeneities are believed to be temporal and spatial variations in microcrack geometry, with variations in crack aspect-ratio one of the major effects (Fig. 3 ). This could lead to variations in (particularly directional) permeability. Since fluid percolation through porous rocks a dominant mechanism in hydrocarbon production in many reservoirs, temporal variations in permeability could substantially modify flow rates. Such variations would be difficult to estimate, difficult to predict, and difficult to assign to a particular cause. The variation of permeability with direction in water-flood operations, for example, shows that 

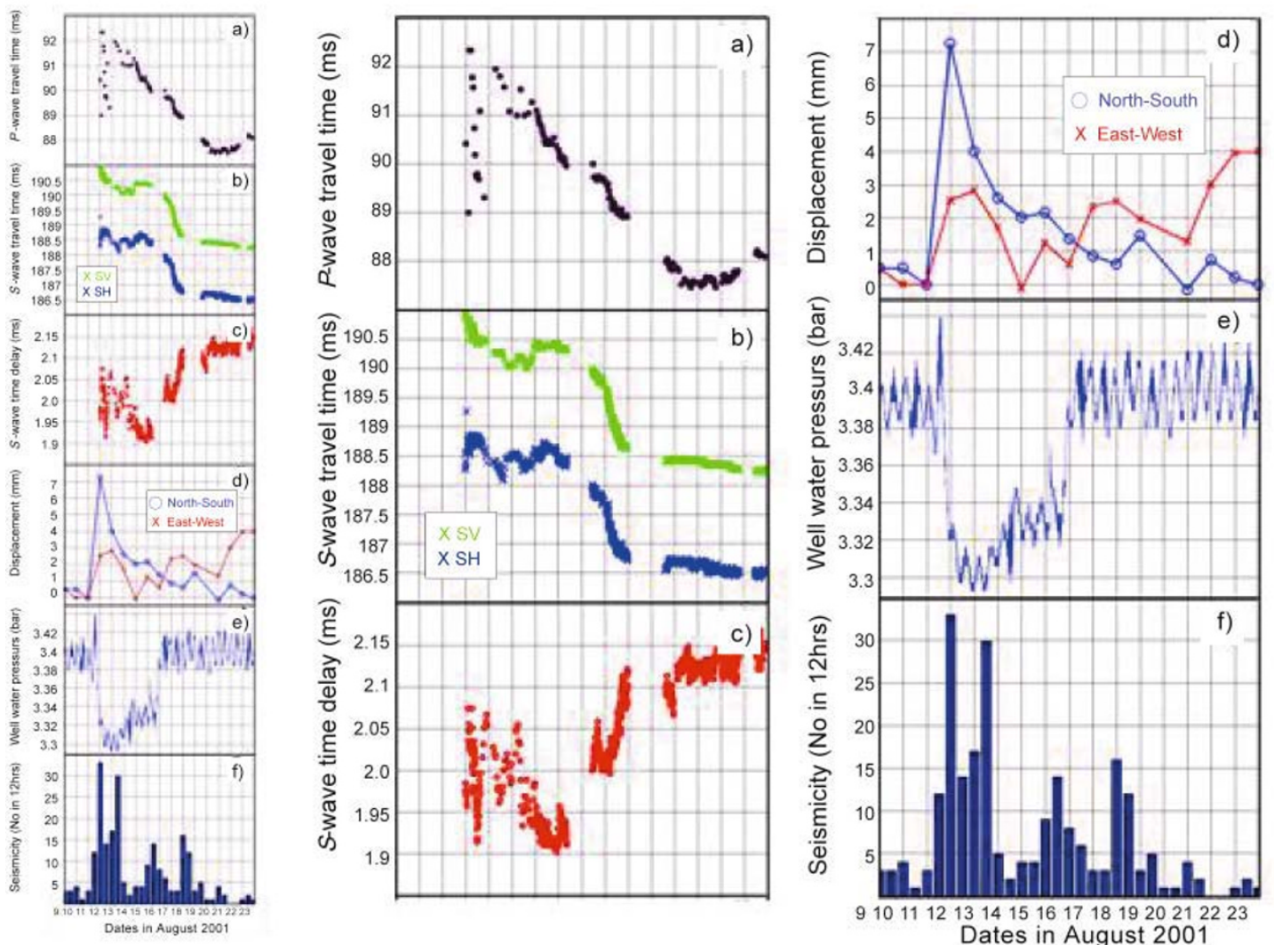

Fig. 5 Observations at the prototype SMS in Northern Iceland (Crampin et al., 2003). (a), (b), and (c) are travel times in $\mathrm{ms}$ of P-, SV- and SH-waves, and SV-SH anisotropy, respectively, at 500m-depth between boreholes $315 \mathrm{~m}$-apart. The directions are parallel to and about $100 \mathrm{~m}$ South of the Húsavík-Flatey Fault (HFF). Shear-waves are polarised $\mathrm{SV}$, and SH propagating in a vertical symmetry direction of the stress field. Also shown are (d) NS and EW (GPS) displacements in $\mathrm{mm}$; and (e) water-pressure 'pulse' measured in bars at $33 \mathrm{~m}$-depth in a well immediately above the HFF (the 'pulse' is $\sim 1 \mathrm{~m}$ deep-drop in water-level; the $\sim 40 \mathrm{~cm}$ oscillations are oceanic tides). Observations (a to e), correlate with (f), a histogram of small scale seismicity on a parallel fault $70 \mathrm{~km}$ NNW of the SMS. The total energy of the seismicity is approximately equivalent to one magnitude M 3.5 event (After Crampin et al, 2003)

aligned cracks have a large affect on hydrocarbon percolation. Thus variations of crack aspect-ratios (the most compliant crack parameter) could have major effects on directional permeability and hydrocarbon production rates. These effects have been demonstrated in observations of various effects on shear-wave splitting above small earthquakes (Appendix).

Taken together these mean that, in detail, rocks in the crust are critical systems of highly sensitive compliant fluidsaturated cracks which can only be imaged by shear-wave splitting. In particular:

1) Detailed seismic measurements are likely to vary with time and space.

2) The longer the seismic ray path, the larger the possible temporal and spatial variations in seismic measurements, because of temporal and spatial heterogeneities.

3) Comparatively minor variations in microcrack geometry could have major effects on porosity and permeability and hence have major effects on hydrocarbon production.

As a consequence conventional seismic observations, where source and/or receivers are surface-based, may suffer possibly severe anomalies in time and space that may not be explicable in terms of conventional geophysics. Meaningful measurements have to be taken at the time and place they are required and need to be recorded as close as possible to the production zone.

\section{Implications of the New Geophysics for hydrocarbon production}

Since liquid-saturated cracks have little effects on $P$-wave propagation, the New Geophysics is likely to have little effect on conventional exploration seismology, where the principal tool is $P$-wave arrival times. Consequently, if one is happy with conventional $P$-wave seismics, and happy with conventional seismic resolution and current production, many of the effects of the New Geophysics can probably be ignored.

However, there are two major effects. New Geophysics potentially alters the behaviour of seismic shear-wave propagation, and alters the geophysical behaviour of the reservoir which could have major effects on reservoir exploitation and recovery.

Three potential applications, amongst many others, are discussed below.

\subsection{Monitoring production with time-lapse seismics by Single-Well Imaging (SWI)}

The movement of oil/water fronts can be potentially 
monitored by differencing (subtracting) record sections before and after some oil field production process in timelapse seismics. Success depends on interpretation of changes in successive records shot with identical source-to-receiver geometry. Such detailed sections are particularly expensive offshore, where many oil fields are situated, and one of the major expenses is laying areal arrays of sea-floor geophones for optimal time-lapse seismics.

Note that the effects of the New Geophysics are nonlinear, as in the $\pm 80 \%$ scatter of shear-wave time-delays observed above all small earthquakes (Crampin, 2004; and Appendix), so that they cannot be interpreted by conventional sub-critical techniques. Consequently, the temporal and spatial effects of the New Geophysics means that any insitu measurements necessarily degrade both temporally and spatially as soon they are made, where the longer the ray paths and the longer the durations the greater the possible degradation.

The only way to avoid such degradation is to make and interpret measurements at the time and place (the producing reservoir) they are required by Single-Well Imaging (SWI) (Crampin, 2006).

The most direct way of recording seismics within a producing reservoir is by time-lapse (4D) single-well imaging (SWI). SWI is where a borehole source (the DOV for example) is repeatedly pulsed, and scattered reflections and diffractions are recorded by three-component geophones in the same borehole as the source. Recordings of a single pulse may well be impossible to interpret. However, if the pulse is repeated before and after there has been some change in the reservoir, Fig. 6 shows that time-lapse (differencing successive recordings) can in principle determine the direction and distance of the changing properties. SWI is a new technique that has several advantages.

1) Time-lapse (4D) SWI can, in principle, locate the direction and distance of any change within the reservoir (the movement of oil-water contacts, for example). Majer et al (1997) recorded SWI changes in $P$-wave reflections from a piezoelectric borehole source on a string of hydrophones, from a known fracture before and after an air injection, recorded in a borehole some $20 \mathrm{~m}$ from the fracture. No shear-waves were recorded.

2) The SWI location of changes (as in Fig. 6) is largely independent of reservoir structure. This means that, in principle, SWI from a single well (ideally the production well) in a producing reservoir could: follow moving oilwater contacts; anticipate water coning; locate bypassed oil (as volumes where there was no fluid movement); locate fractures by SWI location of small acoustic events (and in optimum geometry by direct seismic reflections, as in Majer et al (1997).

3) Analysis (in principle) is simple, automatic, and largely

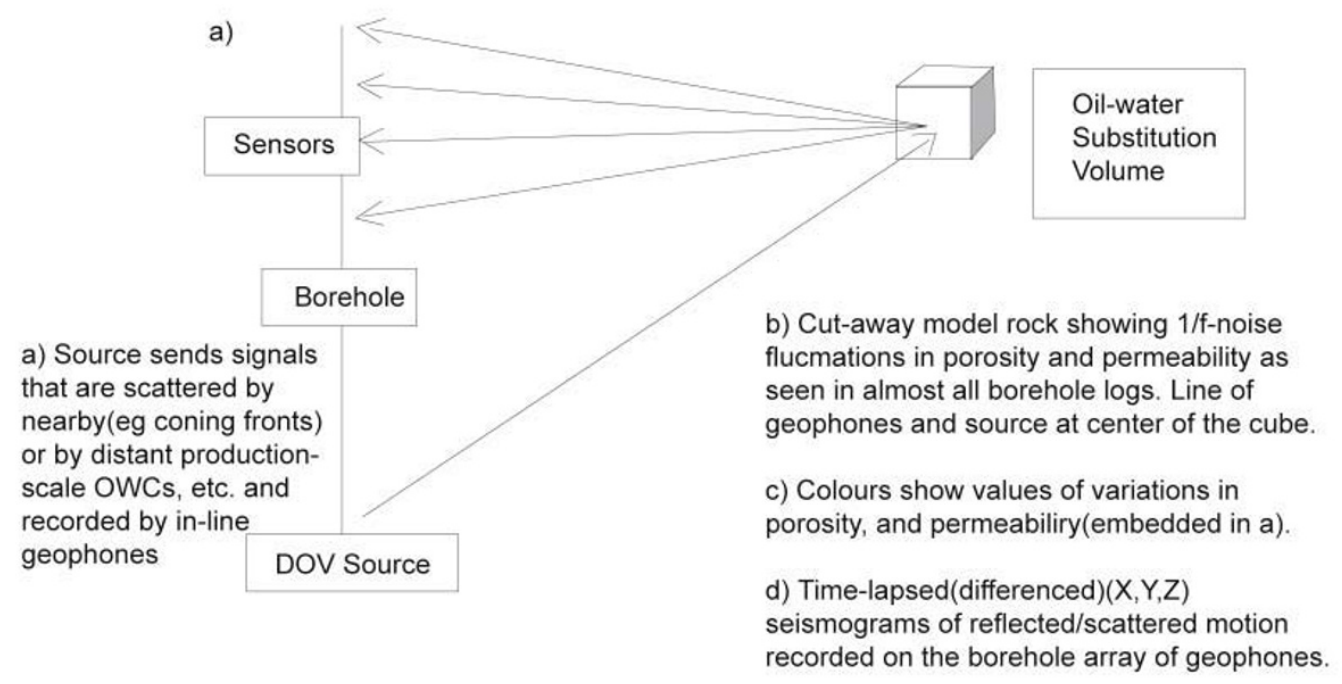

e) Vector interpretation of(d) shows changed parameters averaging about the " $\star$ ".

b)

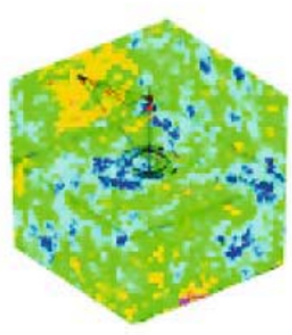

c)

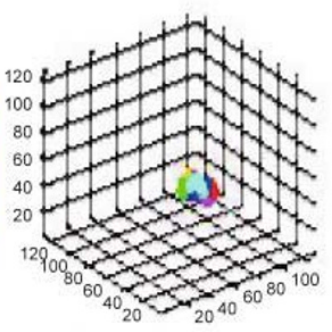

d)

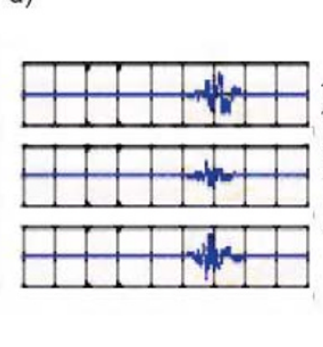

e)

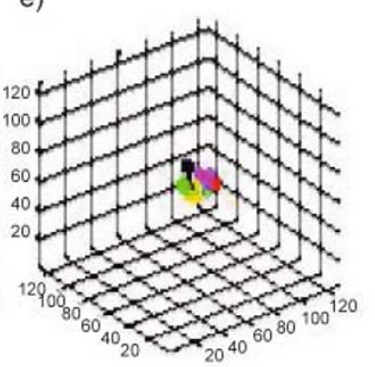

Fig. 6 Modelled time-lapse Single-Well Imaging (SWI) of a cube having observation-based 1/f-noise variations in porosity and permeability before and after small variations located in one corner (cont.) 
independent of structure, thus almost real-time analysis would be possible.

4) There are several significant advantages over alternatives for monitoring production:

(a) SWI would provide the basic parameters for initiating APE-modelling.

(b) SWI operation and processing would be, in principle, automatic and rapid.

(c) SWI is much cheaper $(\mathrm{x} \sim 1 / 100)$ than $4 \mathrm{D}$ reflection surveys, particularly if geophones are located behind the casing or tubulars when first installed.

As a result, SWI opens up alternative techniques for seismic processing, with whole new areas where timelapse seismics could be used for real-time monitoring of the producing reservoir with the possibility of making dynamic changes to optimise production processes during hydrocarbon recovery.

\subsection{Slower production for More Oil REcovery (SMORE)}

It has been demonstrated, above, that the distributions of stress-aligned fluid-saturated microcracks in most in situ rocks are critical systems verging on fracture-criticality and fracturing (Fig. 2). Critical systems impose temporal and spatial cooperative behaviour that has self-similarity and self-organised criticality (SOC) (Davis, 1989; Jensen, 1998). One of the characteristics of SOC is that the self-similarity and calculability only occur when the complex interactions respond to slow (natural) changes (Bruce and Wallace, 1989). Rapid changes and aggressive production strategies are likely to produce chaotic deformation which will not be self-similar or calculable.

Only when slowly driven can the response of a complicated heterogeneous reservoir to specific changes be calculated, or predicted, by APE, as happened in the $\mathrm{CO}_{2}$-injections reported by Angerer et al (2002) in Fig. 4. Stress-relaxation times are not known, and require further investigation. However, preliminary evidence from: i) $\mathrm{CO}_{2}$ injections (Angerer et al, 2002); ii) cross-well seismics in Fig. 5 (Crampin et al, 2003); and iii) changes before earthquakes (Appendix) suggests that the response is comparatively rapid, and that effects could be modelled by APE after only a day or two relaxation - this might be an acceptable delay in oil field procedures for the production of oil, which otherwise would be irrecoverable. The implications are that reservoirs forced by aggressive production strategies will create further heterogeneities, disrupt existing lines of flow that cannot be modelled by APE, and hence yield less oil. This suggests that modelling and calculating the response will only be possible for slow recovery rates, hence the proposal for Slower production for More Oil REcovery (SMORE).

The question is how slow is slow? The delay between the initial and second survey in for Fig. 4 was approximately two weeks and was clearly sufficient as the results were calculable, but it could be much shorter. Note that the slow recovery might need to be for the whole production history. Initial aggressive production is likely to irreversibly disturb the rock, although this not appear to be the case for Fig. 4, which was shot in a mature field.
There are to our knowledge no estimates of how much more is more. Currently, oil fields typically recovery less than $\sim 40 \%$ of the oil in the reserve. Thus, even a conservative increase of $2 \%$, say, to $42 \%$ (it might be substantially greater) of produced oil over possibly marginally longer recovery would be a $\sim 5 \%$ increase in recovered oil. This would be additional profit on the initial infrastructure cost of oil rigs and pipe lines at the cost of a slower recovery rate.

\subsection{Optimising water-flooding by APE-modelling}

The response of the rock mass can be potentially controlled by matching time-lapse shear-wave splitting in 4D reflection surveys or VSPs to APE-modelling with feedback.

This might be important for fluid-injection. If a waterflood operation were initially monitored by appropriate seismic measurements, such as VSPs or SWI, it would be possible to recognise whether cracks had opened in the desired direction to aid fluid sweep operations. If non-optimal orientations were indicated, different injection procedures could be attempted and tested until optimum effects were achieved.

This could establish the likelihood of success of the water fluid operation within hours or days of the flood and long before production returns indicated success or failure.

\section{Conclusions}

Crack-criticality, the New Geophysics, is thought to be a major advance in understanding pre-fracturing deformation. The reason this has not been identified earlier is that the effects depend almost entirely on shear-wave propagation. In the past, seismic investigations have been dominated by $P$-wave propagation, and $P$-waves are rather insensitive to fluid-saturated microcracks (Crampin, 1999). Shearwaves require three-component recording and this is both more expensive to record and more difficult to process than conventional seismic sections of vertical-component geophones or hydrophones.

Since the properties of critical systems are determined by criticality and not by the underlying sub-critical behaviour, we cannot expect to 'understand' the critical behaviour in terms of our experience in conventional sub-critical geophysics. We may be in for some surprises.

The New Geophysics is a recent construct and the full implications will take some time to evolve. Why do we need a New Geophysics? After some $80+$ years of oil production and vast financial investment, we still recover, typically, less than $\sim 40 \%$ of the total oil in the reservoir. We know where there is more oil than we have ever used, in the reservoirs we have abandoned. Why should we be so unsuccessful?

Shear-wave splitting (seismic birefringence) shows there are stress-aligned fluid-saturated microcracks in almost all reservoirs that are so closely spaced they are highly compliant and are consequently critical systems. This means that in detail reservoirs and seismic waves do not behave conventionally. Fig. 4 shows that in situ rocks, including hydrocarbon reservoirs, display sensitivity to minor disturbances, with much greater sensitivity than would be expected in a conventional brittle-elastic crust. 
These and other fundamental anomalies suggest that there is a serious gap in our understanding of how rocks behave. The New Geophysics may not be the whole answer to our lack of success in oil production, but it does provide a new understanding of fluid-rock deformation, where the key observable is stress-aligned shear-wave splitting, and provides the opportunity to: monitor changes with shear-wave splitting; model future behaviour with anisotropic poro-elasticity (APE), predict future behaviour if changing conditions can be quantified, and in appropriate circumstances control future behaviour by feedback.

The crust of the Earth has been shown to be a critical system of closely spaced fluid-saturated microcracks responding with great sensitivity to small disturbances. Such extreme sensitivity verging on criticality is a feature that appears in a huge range of phenomena as suggested in the Introduction. Critical systems are universally common features of complex interactive systems. Consequently, it is not surprising that the rocks in the Earth's crust, like many other Natural phenomena, are critical systems.

If observations of the reservoir are low-resolution, then the New Geophysics can probably be ignored (except for interpreting shear-wave splitting), but if more accurate measurements are attempted, measurements are likely to be less and less understandable as resolution is increased. It is interesting that the practical engineers and managers, to whom this paper is primarily addressed, should have to consider this state-of-the-art physical advance for better appreciation of the behaviour of their reservoirs, and hopefully an increase in oil production.

A New Geophysics has been outlined, suggesting that for detailed understanding of reservoirs and increasing oil production, reservoirs need to be monitored by time-lapse SWI. SWI is a new, largely untried, technology. Fig. 6 indicates that in principle time-lapse SWI works. The most important requirement now is to begin to process real data.

SWI today is in much the same position that reflection seismology was perhaps 70 years ago. There are many known and unknown problems to resolve, as there were with reflection seismology. Since SWI tracking of fluid fronts is largely independent of geological structure, SWI could eventually replace costly reflection surveys. With the massive advances projected in technology and processing, it is suggested that SWI could become a key oil field production tool for the 21 st century.

\section{Acknowledgments}

This work has been supported by many colleagues to numerous to mention individually, but we particularly thank Sheila Peacock for her invaluable contributions. We thank Peter C Leary for the analysis in Fig. 6.

\section{APPENDIX - Brief outline of anisotropy above small earthquakes}

The first confirmed observations of azimuthally-varying shear-wave splitting in the Earth's crust were made in the shear-wave window above a persistent swarm of small earthquakes on the North Anatolian Fault, Turkey, during the Turkish Dilatancy Projects: TDP1, 1979; TDP2, 1980; and TDP3, 1984 (Crampin et al, 1980, Crampin et al, 1985; Evans et al., 1987, respectively). These papers established much of the methodology and terminology for observations of shearwave splitting above earthquakes, and were followed by many observations elsewhere.

Peacock et al (1988) and Crampin et al (1990; 1991) first recognised temporal changes in shear-wave splitting (in retrospect) before the 1986 M 6 North Palm Springs Earthquake in California. These changes in Band-1 of the shear-wave window are believed to be monitoring stressaccumulation before impending earthquakes as modelled by APE. Similar changes have now been observed before (currently) some 15 earthquakes in eight different regions elsewhere.

Fig. A1 shows typical variations in time-delays before the 1998 M 5 earthquake in SW Iceland. The left-hand-side shows an increase in Band-1 time-delays for four months before the earthquake (Crampin et al, 1999; Gao and Crampin, 2004), which can be modelled (Fig. 3) as monitoring the stressaccumulation before the larger earthquake. The right-handside shows a decrease in time-delays starting 4 days before the earthquake (Gao and Crampin, 2004), which is thought to be monitoring the effects of crack coalescence onto the eventual fault break of the larger earthquake (Wu et al, 2006; Gao and Crampin, 2006). Such crack coalescence decreases are observed whenever the source seismicity has sufficient activity to record the decrease.

Logarithms of the duration of the both increases and decreases are (separately) proportional to the earthquake magnitudes (Gao and Crampin, 2004). This means that the times and magnitudes of earthquakes can be stress-forecast from the variations in shear-wave splitting. The time, magnitude, and fault break of the earthquake in the figure was stress-forecast in a comparatively narrow window (Crampin et al, 1999).

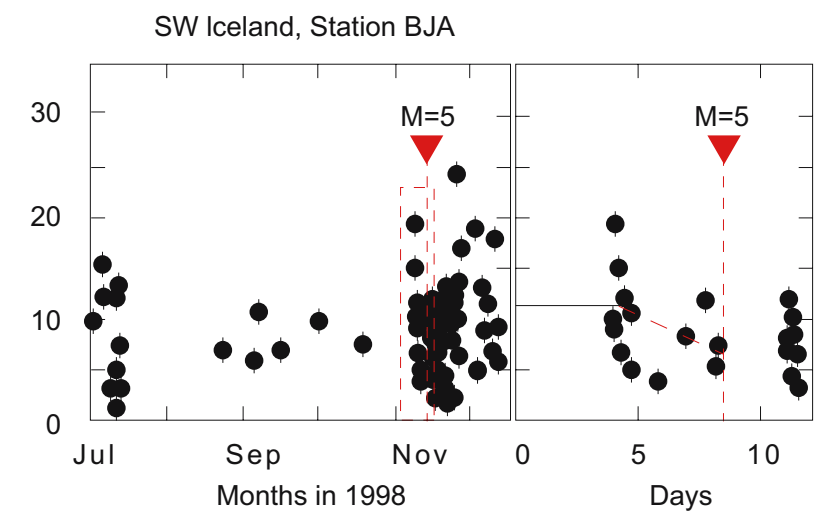

Fig. A1 Variations in time-delays observed above small earthquakes in the shear-wave window at station BJA before the 1998 M 5 earthquake in SW Iceland (Crampin et al., 1999). Time-delays are normalised to $\mathrm{ms} / \mathrm{km}$. Left-hand-side (LHS) shows a least squares line through timedelays (normalise to $\mathrm{ms} / \mathrm{km}$ ) for four months before the $M 5$ earthquake marked with a triangle. Right-hand-side shows the 12 days marked by the dotted box in the LHS with the time-scales with an expanded time-scale. The dashed line is a least-squares fit from where the LHS increase abruptly begins to decrease to the time of the earthquake 
The most extensive investigations of shear-wave splitting have been above a transform zone of the Mid-Atlantic Ridge which runs onshore in SW Iceland, where stress-accumulation was recognised (in retrospect) before some eight earthquakes (Volti and Crampin, 2003a; 2003b; Gao and Crampin, 2006).

Since then, although no further earthquakes have been stress-forecast in real time (successful forecasts required fortuitous combinations of persistent earthquake swarms observed by stations within the shear-wave window and large earthquakes), stress-accumulation has been observed (in retrospect) before some 15 earthquakes worldwide. These range in magnitude from a $\mathrm{M} 1.7$ swarm event in Northern Iceland to the Ms 7.7 Chi-Chi Earthquake in Taiwan. All the phenomena identified in the behaviour of shear-wave splitting above small earthquakes are wholly consistent with the New Geophysics discussed in the main text.

Much of the recent research in shear-wave splitting has been under Protocols of Collaboration between the Institute of Earthquake Science, China Earthquake Administration, Beijing, and the School of GeoSciences, University of Edinburgh.

Much of our knowledge of shear-wave splitting has come from these investigations above small earthquakes. It has been established that the sensitivity of shear-wave splitting to stress-accumulation is a function of the New Geophysics of a crack-critical Earth (Crampin and Peacock, 2005; Crampin, 2006).

\section{References}

*Angerer E, Crampin S, Li X Y, et al. Processing, modelling, and predicting time-lapse effects of overpressured fluid-injection in a fractured reservoir. Geophysical Journal International. 2002. 149: 267-280

Booth D C and Crampin S. Shear-wave polarizations on a curved wavefront at an isotropic free-surface. Geophysical Journal of the Royal Astronomical Society. 1985. 83: 31-45

Bruce A and Wallace D. Critical point phenomena: universal physics at large length scale. In: The New Physics (Edited by Davis P.) Cambridge University Press. 1989: 236-267

Crampin S. A review of wave motion in anisotropic and cracked elasticmedia. Wave Motion. 1981. 3: 343-391

*Crampin S. The fracture criticality of crustal rocks. Geophysical Journal International. 1994. 118: 428-438

*Crampin S. Calculable fluid-rock interactions. Journal of the Geological Society. 1999. 156: 501-514

*Crampin S. Developing stress-monitoring sites using cross-hole seismology to stress-forecast the times and magnitudes of future earthquakes. Tectonophysics. 2001. 338: 233-245

*Crampin S. The New Geophysics: shear-wave splitting provides a window into the crack-critical rock mass. The Leading Edge. 2003. 22: 536-549

*Crampin S. The New Geophysics: implications for hydrocarbon recovery and possible contamination of time-lapse seismics. First Break. 2004. 22: 73-82

*Crampin S. The New Geophysics: a new understanding of fluid-rock deformation. In: Eurock 2006: Multiphysics coupling and long term behaviour in rock mechanics (Edited by Van Cotthem A, Charlier R, Thimus J F and Tshibangu J P) Taylor and Francis, London. 2006. 539-544

*Crampin S and Chastin S. A review of shear-wave splitting in the crack-critical crust. Geophysical Journal International. 2003. 155:
221-240

*Crampin S and Peacock S. A review of shear-wave splitting in the compliant crack-critical anisotropic Earth. Wave Motion. 2005. 41: $59-77$

*Crampin S and Zatsepin S. V. Modelling the compliance of crustal rock: II - response to temporal changes before earthquakes. Geophysical Journal International. 1997. 129: 495-506

Crampin S, Evans R, Üçer B, et al. Observations of dilatancy-induced polarization anomalies and earthquake prediction. Nature. 1980. 286: 874-877

Crampin S, Evans R and Üçer S B. Analysis of records of local earthquakes: the Turkish Dilatancy Projects (TDP1 and TDP2). Geophysical Journal of the Royal Astronomical Society. 1985. 83: 1-16; 17-30; 31-45; 47-60; 61-73; and 75-92

Crampin S, Booth D C, Evans R, et al. Changes in shear wave splitting at Anza near the time of the North Palm Springs Earthquake. Journal of Geophysical Research. 1990. 95, 11: 197-212

Crampin S, Booth D C, Evans R, et al. Comment on "Quantitative Measurements of Shear Wave Polarizations at the Anza Seismic Network, Southern California: Implications for Shear Wave Splitting and Earthquake Prediction" by R. C. Aster, P. M. Shearer \& J. Berger. Journal of Geophysical Research. 1991. 96: 6403-6414

Crampin S, Zatsepin S V, Slater C et al. Abnormal shear-wave polarizations as indicators of pressures and over pressures. 58th Conference European Association of Geophysicists and Engineers. 1996. Amsterdam, Extended Abstracts. X038

*Crampin S, Volti T and Stefánsson R. A successfully stress-forecast earthquake. Geophysical Journal International. 1999. 138: F1-F5

*Crampin S, Volti T, Chastin S et al. Indication of high pore-fluid pressures in a seismically-active fault zone. Geophysical Journal International. 2002. 151: F1-F5

*Crampin S, Chastin S and Gao Y. Shear-wave splitting in a critical crust: III - preliminary report of multi-variable measurements in active tectonics. Issue, Journal of Applied Geophysics. 2003. Special Issue. 54: 265-277

Davis P. The New Physics: A Synthesis. In: The New Physics (Edited by Davis P) Cambridge Univ. Press. 1989. 1-6

Evans R, Beamish D, Crampin S, et al. The Turkish Dilatancy Project (TDP3): multidisciplinary studies of a potential earthquake source region. Geophysical Journal of the Royal Astronomical Society. 1987. 91: 265-286; 287-311; and 313-330

* Gao Y and Crampin S. Observations of stress relaxation before earthquakes. Geophysical Journal International. 2004. 157: 578-582

* Gao Y and Crampin S. A further stress-forecast earthquake (with hindsight), where migration of source earthquakes causes anomalies in shear-wave polarisations. Tectonophysics. 2006. 426: 253-262

Gao Y, Wang P, Zheng S, et al. Temporal changes in shear-wave splitting at an isolated swarm of small earthquakes in 1992 near Dongfang, Hainan Island, Southern China. Geophysical Journal International. 1998. 135: 102-112

Heffer K J, Fox R J, McGill C A, et al. Novel techniques show links between reservoir flow directionality, Earth stress, fault structure and geomechanical changes in mature waterfloods. Society of Petroleum Engineers. SPE Paper 30711. 1995

Jensen H J. Self-organized criticality. Cambridge University Press. 1998.

Leary P C, Walter L A and Crampin S. Physical model for downhole orbital vibrator (DOV) - (1) acoustic radiation. 73rd Annual International Meeting, Soceity of Exploration Geophysics, Dallas. 2003. Expanded Abstract 2: 1532-1535

Majer E L, Peterson J E, Daley T, et al. Fracture detection using crosswell and single well surveys. Geophysics. 1997. 62: 495-504

Peacock S, Crampin S, Booth D C, et al. Shear-wave splitting in the Anza seismic gap, Southern California: temporal variations as possible precursors. Journal of Geophysical Research. 1988. 93: 
3339-3356

Walter L A, Leary P C and Crampin S. Physical model for downhole orbital vibrator $(\mathrm{DOV})-(2)$ seismic radiation. 73rd Annual International Meeting, Soceity of Exploration Geophysics, Dallas. 2003. Expanded Abstract 2: 1536-1539

Winterstein D F, De G P and Meadows M A. Twelve years of vertical birefringence in nine-component VSP data. Geophysics. 2001. 66. $582-597$

*Wu J, Crampin S, Gao Y, Hao P, Volti T and Chen Y T. Smaller source earthquakes and improved measuring techniques allow the largest earthquakes in Iceland to be stress-forecast (with hindsight). Geophysical Journal International. 2006. 166: 1293-1298

*Volti T and Crampin S. A four-year study of shear-wave splitting in Iceland: 1. Background and preliminary analysis. In: New insights into structural interpretation and modelling (Edited by Nieuwland D A). Geological Society London. 2003a. Special Publication. 212: 117-133

*Volti T and Crampin S. A four-year study of shear-wave splitting in Iceland: 2. Temporal changes before earthquakes and volcanic eruptions. In: New insights into structural interpretation and modelling (Edited by Nieuwland D A) Geological Society London. 2003b. Special Publication. 212: 135-149

*Zatsepin S V and Crampin S. Modelling the compliance of crustal rock: I - response of shear-wave splitting to differential stress. Geophysical Journal International. 1997. 129: 477-494

*Available at http://www.geos.ed.ac.uk/homes/scrampin/opinion/.

(Edited by Yang Lei) 